and I therefore offer the following case, not with a view to illustrate the evil effects of a non-development of the ovarian function, from whatever cause that may arise, but rather to bring before the reader one as a type of cases known to be intractable in the highest degree.

CASE. - A young lady, tall, slenderly formed, of childish appearance in face and in person, though fully formed and in no way deficient in intellect, consults a physician under the following circumstances: She complains of constant bad health, vaginal discharges, pain of the back, lassitude, weakness, menstrual flow irregular, scanty, at times interrupted. She passes through several hands and the materia medica is at length exhausted. The physician last consulted recommends marriage, which advice is followed, but her health does not improve, and she dies seemingly consumptive at twenty-seven.

The non-development of the ovaria was probably, in this case, merely a symptom of general bad health, of a feeble constitution, which told on the ovaria as on all the other organs of the body; on the skeleton even, for the pelvis was small and seemingly disproportioned, or too narrow for the stature. She died at twenty-seven, - a critical period in man, but more so, perhaps, in woman. She is then, or ought to be, in the prime of life. To twenty-seven she grows in stature and increases in bulk; from that period she descends in life, having passed that great period in her existence which she can never see again. It is with her, then, a critical period, which she may not pass over unless her hereditary constitution be tolerably sound. - It resembles what thirty-six is in man; he is then in the prime of life, arriving at this culminating point later by nine years than woman. That culminating point with him also is critical and dangerous. To dwell on this would be quite misplaced here. I do not feel disposed to say more on the history of hysteria dependent on orarian irritation extending from thirteen to fifteen; its general character must be well known to all physicians. My object is rather to trace it to its real source; to examine some difficulties, and to excite attention to further inquiry. With a single additional remark I shall, therefore, conclude this part of the section. When puberty appears, when the ovaries enter fully on their action, the young person, from being thin, lean, and unattractive, without form or beauty, may become, in a few weeks or months, a person of transcendent beauty and of the grandest form, full of grace and unaffected dignity; prudent, tender, affectionate, thoughtful, - perfect already in mind and in body. To bring this about, the original proportions, not merely of all the features to each other, but also of the great divisions of the torso to its respective parts and to the limbs, and of these latter to the torso, are modified. When these original proportions, over which no ovarian development can exercise the smallest influence, are good, the beanty then of the individual may assume, and does assume, the highest order, and the being become for a time almost perfect.

But to sustain these noble forms for a few years, nay, even for a few months, seems more than the natural constitution in most women is equal to. Latent germs of disease, hereditary or otherwise, are called into play, against which the terms of perfect development may struggle in her case in vain. The axillary glands swell, cutaneous disease appears, the nose and mouth enlarge, and the features alter exceedingly; all this may happen quite independently of marriage. But these are changes, the tracing of which belongs rather to the physiologist than the physician; and resigning them to his consideration, I proceed next to trace rapidly the history of that most serious of all maladies-namely, mental alienation, examining into its history as a consequence and a result of disturbed, diseased, and impeded ovarian action. This the most lamentable of all the morbid sympathies, originating, as it frequently aoes, in diseased ovaria, in disturbed function of these all-important organs, of impeded development by forced celibacy, occasionally occurring even in the married, and then probably due to an excessive activity in their action; this calamity, when it assumes the terrible form of mania, disturbing all the perceptions, judgments, and actions of the individual, may be traced, as we shall find, to the ovaria in cases wherein hitherto these organs had been in no way suspected. But I am reminded by many cases which at this moment occur to me, that this very disease, mania, is occasionally preceded by epilepsy, or at least by epileptic forms of hysteria. Let me briefly, therefore, before concluding this section, consider the epileptic form of hysteria arising from ovarian disease, ovarian irritation, ovarian excitement, and which no doubt has so frequently been mistaken for true epilepsy.

Of the Protean form of hysteria I had thought all practical men were sufficiently aware. How it puts on, imitating to the life, nearly all other forms of disease, from the simply hysterical laugh to the all but maniacal scream. Thus it imitates trismus, hæmoptysis, pleurisy, pleurodynia; diseases of the heart, of the liver, of the joints, of the brain; hepatitis, peritonitis, hysteria, pericranitis, dyspepsi.., gastrodynia, epilepsy. I had thought that all physicians had known this well ; but a suspicion arises in my mind, at least in regard to the last of its imitations, epilepsy, that this form at least of its multitudinous imitations is not well understood. The hysteric form of epilepsy arising from ovarian irritation is simply hysteria: it is not epilepsy. When engrafted on a feeble constitution, liable to head affections, hereditary or otherwise, it may in some rather rare cases become decided epilepsy, but this is undoubtedly a rare occurrence. True epilepsy is not a common disease in woman ; and to consider and treat the hysteric epilepsy originating in ovarian irritation as true epilepsy is to mislead the profession and the public, and to confound diseases diametrically opposed in their nature, and to injure true pathology.

True epilepsy is but too often of hereditary origin; it springs from disease or diseased functions of the cerebro-spinal axis; its course is from the centre towards the circumference; it can seldom originate in any radiation of diseased sensation from the periphery to the centre; lastly, when the case is carefully observed, it can scarcely be mistaken for the hysterical form of epilepsy, or what may be termed hysterical, epileptic convulsions. Convulsions, unhappily, we think, called epileptic, occur in pregnant women, and are supposed to originate in toxæmia, meaning, by the new-invented term, poisoning of the general mass of blood by means of the lochia or by organic disease of the kidney cansed by the pressure of the pregnant uterus, or simply by pressure on the renal veins, independent of any organic disease.

These hypotheses, for they are merely such, may or may not be true; future extended experience can alone decide this point; in the meantime, it is to be regretted that such convulsions are called epilepsy. It is also still rather problematical to view the osteophytic spicula deposited on the inner table of the skull (frontal bone) as being a frequent cause of epilepsy or of epileptic convulsions, seeing that such spicula occur, I believe, in many persons who have not been known to labour under such disorders. Ovarian irritation is not, then, a frequent cause of epilepsy, perhaps never as a cause but in the predis. posed. It would seem, according to the views of Rokitansky, that there is at each period of pregnancy an osseous deposit on the inner surface of the cranium, and that this, in fact, is a normal condition. Now, we doubt the fact, if it be meant to be universally applied, though admitting its occasional occurrence. As to the "peripheral extremities of the intra-cranial nerves," I know nothing of them, but shall be glad to admit their existence when demonstrated.

The intra-cranial osteophytes, then, of pregnant women have still to be proved to be a normal condition. They vary, as it is well known, in extent and amount; when numerous, projecting irregularly inwards, and pressing on the dura mater, they may prove a cause of true epilepsy, or even of convulsions assuming an epileptic character. But this subject requires a much more extended inquiry than has yet been given to it. The toxæmic theory of puerperal convulsions is contradicted by the plainest facts.

\section{ON THE COMPOSITTON OF BREAD.}

BY WILLTAM ODLING, M.B., F.C.S., PROFESSOR OF PRACTICAL CHEMISTRY AT GLY'S HOSPITAI.

Is fulfilment of the duties of my situation as Medical Officer of Health for the parish of Lambeth, I have recently directed my attention to the quality of the bread supplied to my fellowparishioners. I have examined twenty-five specimens, each procured from a different baker. Until the completion of the entire series of experiments, I was ignorant of the prices of the specimens and of the shops at which they were purchased. The loaves were two-pound loaves, obtained new--that is to say, during the day on which they were baked. The top crust of each loaf was sliced off, and then a layer about two inches thick removed, trimmed at the edges, and submitted at once to examination. The results are as follows:-

Water. - The two highest determinations of water indicated 46.71 and 45.42 per cent. respectively; the two lowest determinations gave $38 \cdot 62$ and 41.06 per cent. respectively; the mean of the twenty five determinations gave 43.83 per cent 
the average amount of water existing in the crumb of new bread.

Saline matter. - The two highest determinations of the amount of ash yielded by the dry bread were $2 \cdot 90$ and $2 \cdot 62$ per cent. respectively; the two lowest determinations gave 1.54 and 1.87 per cent. respectively; the mean of the twentyfive determinations gave $2 \cdot 30$ per cent. as the average amount of ash in dry bread, or 1.30 per cent. in freshly-baked bread. The average amount of dry substance in bread being, according to my experiments, $56 \cdot 17$ per cent., a two-pound loaf would yield 7863.8 grains of dry substance which, calculating from my average, would give 180.8 grains of ash. Inasmuch as dry flour yields, on the average, 1 per cent. of ash, 7863.8 grains of flour would yield $78 \cdot 6$ grains of ash, which, being subtracted from the 180.8 grains, yielded by the two-pound loaf, would leave 102.2 grains of introduced saline matter consisting principally of common salt and alum.

Nitrogen or Gluten. - The prevalent opinion amongst physiologists is, that the nutritive properties of bread are in proportion to the amount of its nitrogenized constituents, though, indeed, the correctness of this opinion has been called in question by two eminent agricultural chemists, Messrs. Lawes and Gilbert. The two highest determinations of nitrogen that I have obtained are respectively 3.42 and 3.21 per cent. in the dried, or 1.89 and 1.83 per cent. in the freshly-baked bread the two lowest determinations are 1.66 and 1.81 per cent. in the dried, or 0.93 or 1.01 per cent. in the freshly-baked bread. It is observable that the highest determination is more than double the lowest-a circumstance by which I was at first much surprised. On referring, however, to published analyses of flour, I find that the amount of gluten varies from 7 to 15 per cent. The mean of all the twrenty-five estimations gives $2 \cdot 23$ per cent. of nitrogen in the dry, and $1 \cdot 26$ per cent. in the freshly-baked bread. My average of nitrogen in freshly-baked bread corresponds very closely with the prior determinations of Dr. Playfair, and of Messrs. Lawes and Gilbert.

Alum.- - Alum was detected in eighteen out of the twentyfire samples. No estimation was made of the quantity; but, from the appearance of the precipitates, I should distinguish three loaves as containing very much, and three as containing very little, of this impurity. The very extended use of alum in bread, is in itself almost an evidence that some benefit accrues to the baker from its employment. The probability of its exerting an injurious effect upon the consumer, must depend materially upon the quantity introduced. Whatever may be our opinions upon the subject, however, we have, it must be confessed, no positive evidence that the habitual use of alum in bread is, or is not, deleterious.

The qualities attributed to alum by different bakers vary considerably. I believe that one great effect of alum is to hinder the transformation of starch into sugar, during the process of baking. Two years ago, I had sent to me for analysis, a loaf which was sticky, saccharine, and sodden throughout, but which had been made from apparently good flour. In the autumn of last year, I received from Tring, in Hertfordshire, a sample of flour which was unadulterated, which contained the normal quantities of gluten, starch, dextrin, \&c., each of good quality, which had, in fact, only one fault-it would not make bread. The result of the baking was a sweet, sticky, dark-coloured mass. The wheat from which these two flours had been prepared, had, doubtless, undergone a partial malting, with the consequent formation of diastase. Between such specimens as these, and the most perfect fiours, every grada tion exists. The object of the baker being to retain as much of the starch as possible, with its chemical and physical properties unaltered, he probably finds this object best attained by the addition of alum, which substance $I$ have found experimentally to interfere greatly with the transformative powers of diastase. Of the seven samples of bread which did not contain alum, two were cheap loaves obtained from the bakehouses of two large associations of workpeople, and four were fullpriced loaves purchased at old-established shops of high repute.

COPPER - In seven out of the twenty-five samples, the presence of copper was detected. These are, I believe, the first cases on record in which English bread has been shown to contain copper. I should be loth, however, to charge London bakers with the intentional sophistication of their bread with blue vitriol, unless it could be clearly proved that the presence of copper was otherwise inexplicable, or unless we had positive evidence of the fraudulent introduction of the cupric salt. The probabilities in favour of the accidental presence of the copper are-1st. That in three of the samples containing copper, no alum was detected. Those who would adulterate with copper would probably adulterate with alum also. In two of the above cases, however, the copper was in the most minute traces. 2ndly. That copper is a very widely distribated element, and that it might possibly, though somewhat improbably, have been introduced in the salt, alum, yeast, \&c., or have resulted from the use of copper utensils. The probabilities in favour of the fradulent introduction of the copper are1st. That sulphate of copper has the reputation, probably well founded, of exerting a powerful effect in facilitating the fermentation, improving the appearance, and increasing the water-retaining powers of bread. The specimen of bread which yielded me the highest per-centage of water contained copper. 2ndly. That in four of the specimens, at any rate, three of them containing alum in addition, the amount of copper was sufficient to effect its ascribed improvements.

The experiments upon which the above observations are founded were performed as follows:-The nitrogen was determined by a slight variation of Will and Varrentrap's method, as modified by Peligot. This consists in a combustion of the bread with soda-lime, and an estimation of the amount of ammonia produced, by means of standard solutions. For the detection of the alum, about 500 grains of bread were incinerated. To the resulting ash, weighing generally about six or seven grains, two or three drops of chlorhydric acid, with a few drops of water, were added. After a short ebullition, an excess of solution of potash, usually amounting altogether to five or six drops, was added. The filtrate was then acidified with chlorhydric acid, and slightly supersaturated with ammonia, whereby the alumina was precipitated. The potash had been purified by solution in alcohol prior to its solution in water. From an ounce of its aqueous solution, a trace of alumina could be precipitated, but the quantities of solution used in the above experiments gave no indication whatever of impurity. The advantages of the above-described process are, that the quantity of the reagents is reduced to a minimum, and that the whole of the alumina existing in 500 grains of bread is concentrated in less than a drachm of liquid. Aqueous infusions of each of the alumed breads had markedly acid re. actions, probably from bisulphate of potash, and yielded considerable precipitates of sulphate of baryta. For the detection of the copper, about 500 grains of bread were incinerated to a white ash. This was treated with chlorhydric acid in a small platinum capsule, and then an electrolytic action established by the introduction of a piece of zinc, so as to touch the platinum through the liquid. The metallic deposit on the platinum, when treated with a drop of ammonia, yielded a blue liquid, which, after neutralization, gave a decided chocolate-coloured precipitate with ferrocyanide of potassium. The metallic deposit, the blue solution, and the red precipitate were obtained in each of the seven cases.

Addendum. - Since writing the above article, I have again obtained bread from five ont of the seven bakers whose loaves previously yielded me copper, and in each instance have again detected the impurity.

February, 1857.

\section{ON A}

\section{CASE OF OVARIAN DISEASE :}

\section{OVARIOTOMYY DEATH ON THE THIRD DAY FROM DESTRUCTION} OF THE BRONCHIAL MUCOUS SURFACE.

By C. BLACK, M.D. Lond., F.R.C.S., \&c. (Concluded from $p$. 111.)

Sectio cadaveris nine hours after death.-Rigor mortis slightly present; abdomen somewhat tympanitic; edges of the wound adherent throughout their extent. On removing the sutures, they did not separate in the slightest degree, and it required considerable force to break up the union which had already taken place. The peritoneum, to the extent of half an inch on each side of the line of incision, was of a faint, rosy blush; whilst the general peritoneal surface was as free from inflammation as on the day of operation. The pedicle ligature was surrounded by a copious quantity of apparently healthy plastic exudation, and was found to be very firmly tied. The omen: tnm, elongated by the growth of the tumour to which it was attached in two places, was adhering, by its lowest point, to the exudation thrown out around the pedicle. The left ovary was healthy, but in the corresponding broad ligamert of the uterus, 